\title{
Argo剖面浮标显示的棉兰老岛以东中层环流的 中尺度信号特征
}

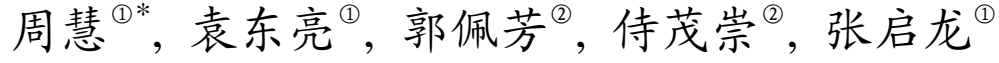 \\ (1) 中国科学院海洋研究所海洋环流与波动重点实验室, 青岛 266071; \\ (2) 中国海洋大学物理海洋实验室, 青岛 266071 \\ *E-mail: zhouhui@qdio.ac.cn
}

收稿日期: 2008-11-04; 接受日期: 2009-06-15

国家自然科学基金(批准号: 40806010)、国家自然科学基金重大项目(批准号: 40890151)、国家重点基础研究发展计划(编号: 2007CB816002, 2006CB403603)、中国科学院知识创新工程重点项目(编号：KZCX2-YW-218, KZCX2-YW-214)、国家杰出青年基金项目(批准号： 40888001)、山东省杰出青年基金和中国科学院“百人计划”资助

\begin{abstract}
摘要 利用 Argo 剖面浮标的轨迹资料研究了棉兰老岛以东中层(1000 2000 m?)深度上的 中尺度环流特征. 浮标漂移深度的轨迹表明, 棉兰老岛以东的中层环流包含非常显著的中尺 度信号特征。浮标的漂移流速表明了该区域的气旋式涡旋和反气旋式涡旋在 $1000 \mathrm{~m}$ 深度上 的平均切向速度约为 $20 \mathrm{~cm} \cdot \mathrm{s}^{-1}$, 随着深度增加速度有所降低, 在 $2000 \mathrm{~m}$ 深度上流速约为 10 $\mathrm{cm} \cdot \mathrm{s}^{-1}$. 以上结果暗示在棉兰老岛以东区域, 依据零速度参考面的动力计算可能存在着较大 误差. 涡旋的平均涡度和赤道 $\beta$ 平面罗斯贝数表明, 这些中尺度涡旋对于棉兰老岛以东中层 环流的质量和涡度平衡起着重要的作用.
\end{abstract}

关键词

棉兰老岛

中层环流

中尺度涡旋

Argo 剖面浮标
棉兰老岛是菲律宾群岛中最南端的一个大岛(图 1). 由于棉兰老岛以东海域是热带西太平洋几种重要 水团的交汇区, 因此, 该区域的环流对于局地以及整 个太平洋海盆尺度的质量和热量平衡都起着非常重 要的作用 ${ }^{[1 \sim 3]}$. 观测表明, 北赤道流(NEC) 将太平洋 表层水体输送到菲律宾群岛以东沿岸, 而后分别由 黑潮和棉兰老流(MC) 向北和向南输送 ${ }^{[1 ~ 3]}$. 在北半球 夏季, 新几内亚沿岸流将南太平洋热带水体输运到 该区域, 新几内亚沿岸潜流则全年都将南极中层水 从南半球输运到此 ${ }^{[4]}$. 由 $\mathrm{MC}$ 携带的水体以及源自南 半球的水体又被北赤道逆流(NECC) 向东输送. 在 NECC 南北两侧分别存在着哈目黑拉涡(中心位于 $4^{\circ} \mathrm{N}, 130^{\circ} \mathrm{E}$ 附近)和棉兰老浴(中心位于 $7^{\circ} \mathrm{N}, 128^{\circ} \mathrm{E}$ 附
近 $)^{[5]}$.

目前，对于棉兰老岛以东海域的次表层环流的 认识还比较匮乏, $300 \mathrm{~m}$ 之下的环流信息主要是通过 地转计算得到的. $\mathrm{Hu}$ 等 ${ }^{[6]}$ 根据沿着 $7.5^{\circ} \mathrm{N}$ 的两条水文 断面的观测指出, 在棉兰老流下面可能存在着多个 次表层北向流核, 有些可以从 $200 \mathrm{~m}$ 延伸至 $1000 \mathrm{~m}$ 深且通常位于 $\mathrm{MC}$ 的离岸一侧下方, 遂将其命名为棉 兰老潜流(MUC). Lukas 等 ${ }^{[2]}$ 在一次水文调查中也证 实了 MUC 的存在. 但是, 单独的断面调查所估算的 流速可能会因为内潮或瞬时流等非地转扰动而产生 混淆信号. 进一步的研究表明 ${ }^{[7]}$, 该次表层北向流在 不同航次变化非常大, 它可能是该区域再循环的一 部分 ${ }^{[8]}$. 在 20 世纪 80 年代中美联合调查的 8 个航

引用格式: Zhou H, Yuan D L, Guo P F, et al. Meso-scale circulation at the intermediate-depth east of Mindanao observed by Argo profiling floats. Sci China Earth Sci, 2010, doi: 10.1007/s11430-009-0196-7 


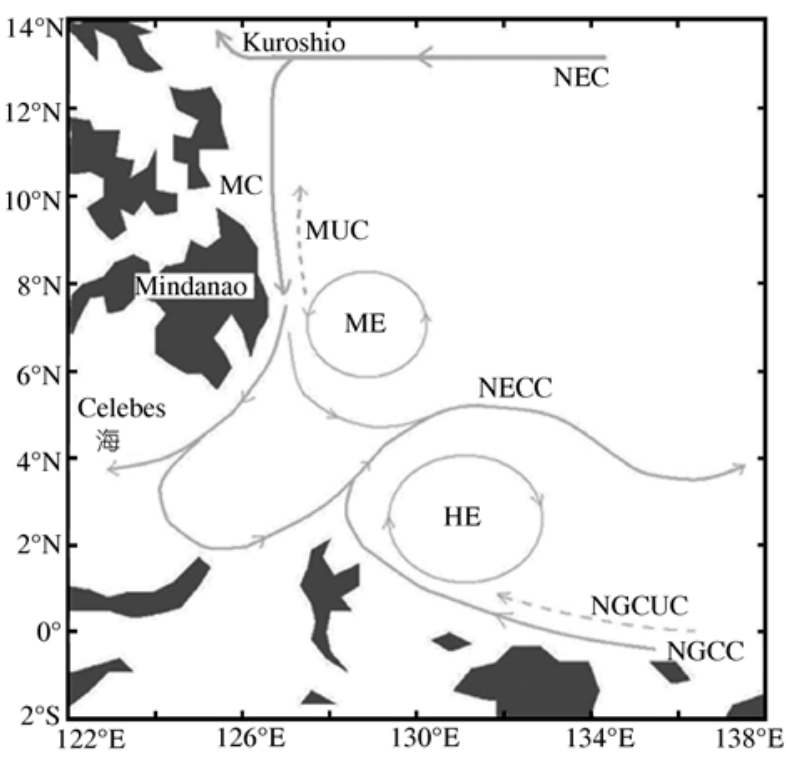

图 1 棉兰老岛与新几内亚岛之间的流态示意图

实线代表表层及次表层海流, 例如棉兰老流 $(\mathrm{MC})$, 北赤道逆流 (NECC), 新几内亚沿岸流(NGCC, 夏季流向), 棉兰老浴(ME)和哈 目黑拉浴(HE). 细线代表次表层至中层海流, 如棉兰老潜流(MUC) 和新几内亚沿岸潜流(NGCUC)

次的调查中, 该次表层流核均不清晰 ${ }^{[9]}$, 在 1988 和 1989 年 4 月份的两次调查中甚至根本不存在北向的 次表层流(见 Wang 和 $\mathrm{Hu}^{[7]}$ 中图 $1(\mathrm{~g}$ )和(h)). Firing 等 ${ }^{[10]}$ 通过直接的 ADCP 流速观测指出该区域次表层 环流的时空变化非常大, 且表现为一系列的中尺度 浴旋. 模式结果也显示该区域的浴旋活动非常活 跃 $^{[11,12]}$. 但是, ADCP 只能观测 $500 \mathrm{~m}$ 以上环流速度, 对于 $500 \mathrm{~m}$ 以下的该区域中层的中尺度浴旋结构至 今尚无连续的跟踪观测.

本文将利用该区域的 Argo 剖面浮标的轨迹资料 来估算浮标漂移深度的流速, 并据此研究棉兰老岛 以东海域中层环流的中尺度信号特征. 由于 Argo 资 料可以对海流进行直接的拉格朗日观测并提供该区 域次表层环流的连续轨迹, 所以与传统的地转流计 算相比具有明显的优势.

\section{1 数据和方法}

\section{1 数据}

本文所采用的数据来自国际 Argo 计划全球数据 分发中心(Global Data Assembly Centers: ftp://usgodael.fnmoc.navy.mil/pub/outgoing/argo/). 该数据集提
供 Argo 剖面浮标观测的剖面数据、元数据、轨迹数 据以及技术数据. 这些数据均源自始于 1998 年的国 际 Argo 计划, 该计划旨在较大空间尺度上进行全球 海洋中的温度和盐度观测. Argo 剖面浮标通常每隔 $10 \mathrm{~d}$ 左右从其漂移深度(通常是 1000 2000 m)上升到 海面，并在上升过程中进行温度、盐度观测. 除了可以 进行温度、盐度剖面的观测, Argo 浮标还可以通过卫 星定位来观测表层流速和漂移深度上的流速. 本文所 使用的 Argo 数据已经由中国 Argo 实时资料中心 (http://www.argo.org.cn/) 按照 Argo 资料用户手册的要 求进行了质量控制. 另外, 本文还进行了浮标定位精 度、传感器校准、压力测试等质量控制, 详见 Xie 等 ${ }^{[13]}$.

\section{2 方法以及流速估算介绍}

一个 Argo 剖面浮标的一次观测循环可分为 4 个 工作阶段：上升、表层漂移、下潜和深层漂移. 温度 和盐度剖面的观测通常是在浮标从漂移深度上升到 海面的过程中进行的. 然后, 浮标在海面漂移 7 25 h, 向经过其上空的 Argos 通讯卫星发送采集到的信息, 通过卫星将观测数据传送到地面接收站, 地面接收 站完成对浮标的定位. Argos 卫星对浮标进行定位, 通常在 5 20 次之间, 且间隔不等(从几分钟到几个小 时), 主要取决于卫星的繁忙程度. 利用这些表层定 位信息就可以估算表层流速和漂移深度的流速. 完 成表层定位以及数据传输后, 浮标就会自动下潜到 漂移深度并随着该深度上的海流漂移 $10 \mathrm{~d}$ 左右，而 后自动上升到海面重复上述过程. 当浮标在下潜、上 升和水下漂移时没有数据实时传送给地面接收站.

但是根据 Argos 卫星系统记录的浮标定位信息, 是可以估算出漂移深度处的流速的. 目前, 利用浮标 定位信息估算中层流速的误差估算已经开展了许多 工作 ${ }^{[13 \sim 15]}$. 本文中我们采用了 Park 等 ${ }^{[15]}$ 提出的一种 既简单、精度又高的方法来估算中层流速. 该方法假 设浮标在海面的轨迹是线性运动和惯性运动的合成, 这两项都可以通过浮标在表层的定位信息采用最小 二乘法计算出来. 浮标在下潜和露出水面时的表层 位置信息可以根据压力测量由观测到的浮标位置信 息外插得到. 这样，根据浮标在下潜和露出水面时的 距离就可以估算由中层海流引起的浮标的漂移速度. 但是，该方法并未考虑表层和漂移深度之间的地转 剪切. Park 等 ${ }^{[15]}$ 估算了浮标在上升和下潜过程中由垂 向速度剪切引起的位移并指出该方法得到的漂移深 
度的流速误差约为 $0.2 \mathrm{~cm} \cdot \mathrm{s}^{-1}$.

图 2 给出了 5900224 号浮标(a)和 2900516 号浮 标(b)在浮出水面后的第一个定位和下潜之前的最后 一个定位之间的位移, 该位移反映了浮标在海面上 漂移期间由于表层海流引起的位移. 由于 2900516 号 浮标有些数据缺失, 所以其轨迹不是连续的. 从图中 我们可以看出, 浮标第一次定位构成的轨迹和最后 一次定位构成的轨迹是非常一致的, 这说明表层流 对浮标在中层漂移的影响是比较小的. 本文中其他 浮标的轨迹也表明了这一点. 另外, ARGO 浮标的漂 移轨迹还可以通过 RAFOS 浮标的运动路径得到佐证. RAFOS 浮标沿着等压面运动, 其轨迹由深海声呐定 位, 可以很好地观测其漂移深度上的海流. 因此, Machín 等 ${ }^{[16]}$ 比较了沿着中大西洋海脊的深层西边 界流区在同一时间、同一位置、同一深度上释放的 RAFOS 浮标和 Argo 剖面浮标的运动轨迹, 估算了由 Argo 浮标轨迹计算的中层海流误差. 他们的比较结 果表明, 两种测量在统计意义上是等同的, 根据 RAFOS 浮标轨迹估算的速度与 Argo 剖面浮标得到 的流速基本是一致的, 均在误差范围内(西边界流区

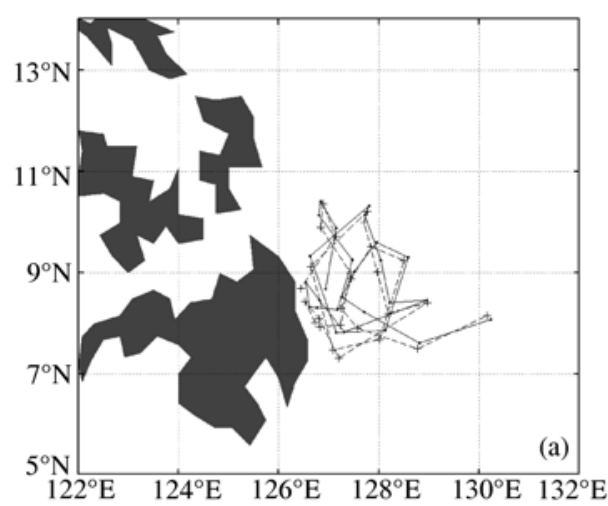

该误差约为 $12 \mathrm{~cm} \cdot \mathrm{s}^{-1}$ ), 因此, 本文中根据 $\operatorname{Argo}$ 剖面 浮标轨迹反演到的中层流是可信的.

\section{2 浮标轨迹}

截止到 2007 年 6 月 30 日，已有 27 个 Argo 剖面 浮标被投放到棉兰老岛以东区域或者漂移至此. 其 中，大部分浮标离棉兰老沿岸较远或者仅仅偶然经 过该区域，这些浮标不能很好地反映出棉兰老岛以 东海域的中层西边界流结构. 因此, 图 3 中仅仅显示 了那些进入 $129^{\circ} \mathrm{E}$ 以西, $12^{\circ} \mathrm{N}$ 以南区域且停留时间较 长的浮标轨迹. 就像上文中所提到的, 这些轨迹主要 代表了浮标在漂移深度上的运动. 从图 3 中可以看出, 没有一个浮标从棉兰老岛以东跨过北赤道流向北运 动或者从北赤道流下面向东运动. 这其中有 3 个浮标 (5900224, 5900131 和 2900516 号浮标)在棉兰老岛以 东停留了较长时间, 而且他们的轨迹反映出了该区 域的中尺度环流形态(图 3). 这 3 个浮标在其工作的 34 个月期间共观测到了 126 个剖面, 其观测各参数 见表 1. 我们将通过分析这 3 个浮标的运动轨迹来研 究棉兰老岛以东海域的中层环流结构.

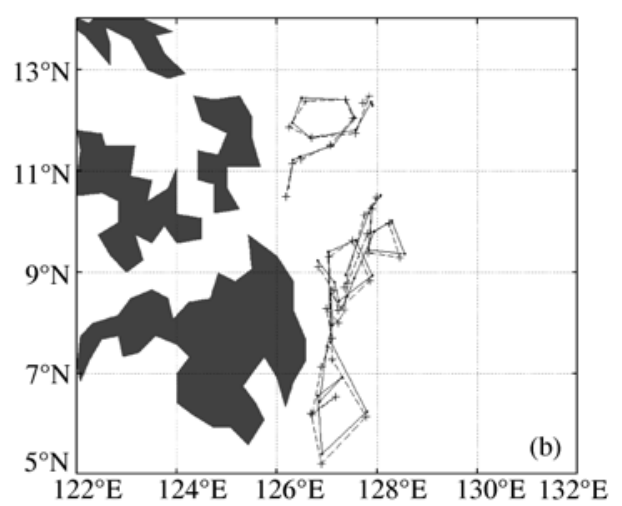

图 25900224 号浮标轨迹(a)和 2900516 号浮标轨迹(b)及各自的第一次定位位置(•)以及最后一次定位位置 $(+)$

表 1 Argo 剖面浮标观测参数

\begin{tabular}{cccc}
\hline WMO ID & 5900224 & 5900131 & 2900516 \\
\hline 下水日期 & $2003-01$ & $2002-01$ & $2005-10$ \\
纬度 & $8.7^{\circ} \mathrm{N}$ & $11^{\circ} \mathrm{N}$ & $12.76^{\circ} \mathrm{N}$ \\
经度 & $129.9^{\circ} \mathrm{E}$ & $145.3^{\circ} \mathrm{E}$ & $133.6^{\circ} \mathrm{E}$ \\
开始日期 & $2003-01-14$ & $2002-02-23$ & $2005-10-22$ \\
结束日期 & $2003-10-08$ & $2003-10-04$ & - \\
上升间隔 $/ \mathrm{d}$ & 10 & 10 & 10 \\
压力/dbar & 1500 & 1000 & 2000 \\
最大压力/dbar & 2025 & 1038 & 2000 \\
\hline
\end{tabular}



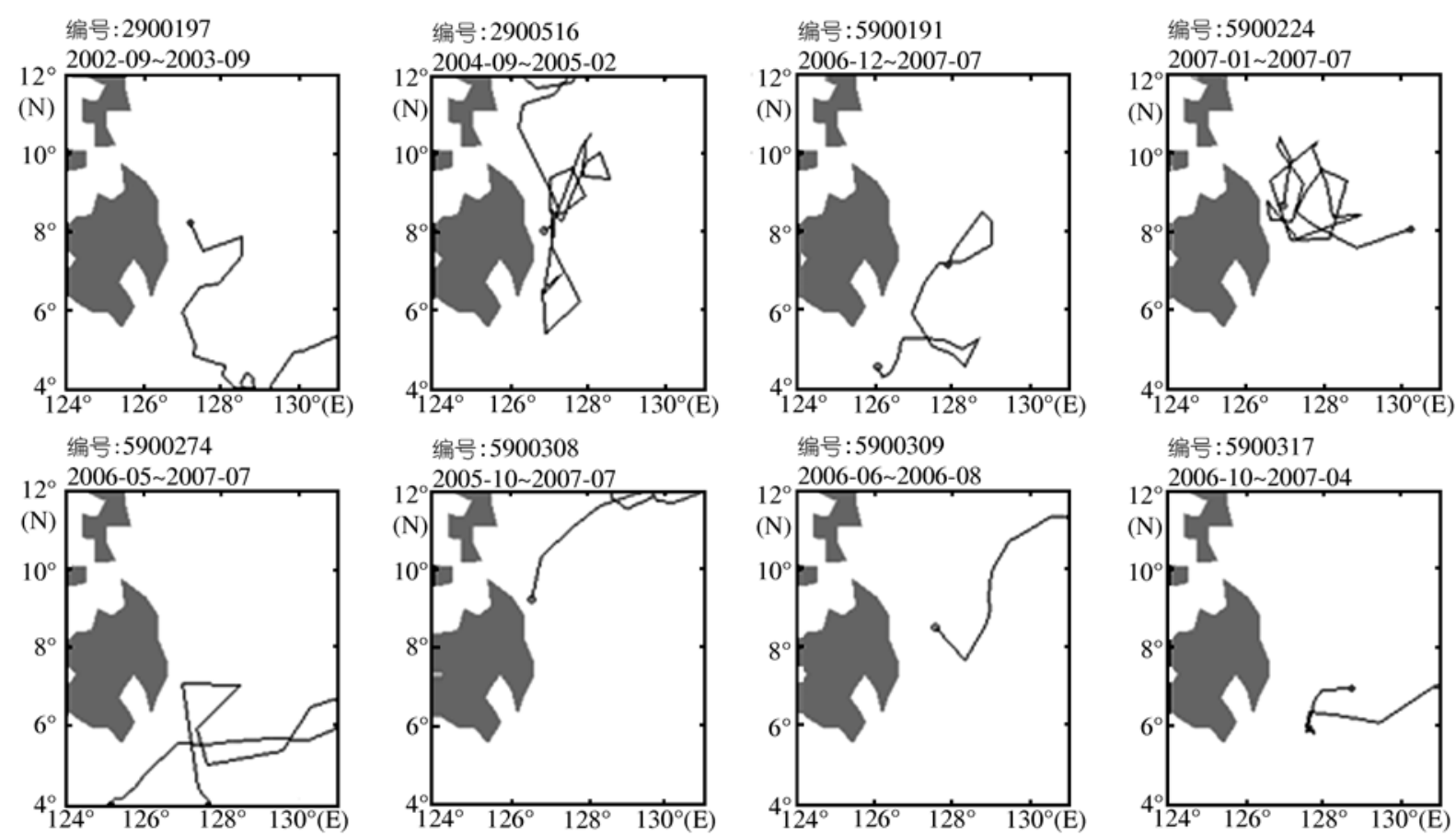

$$
\text { 编号:5900317 }
$$
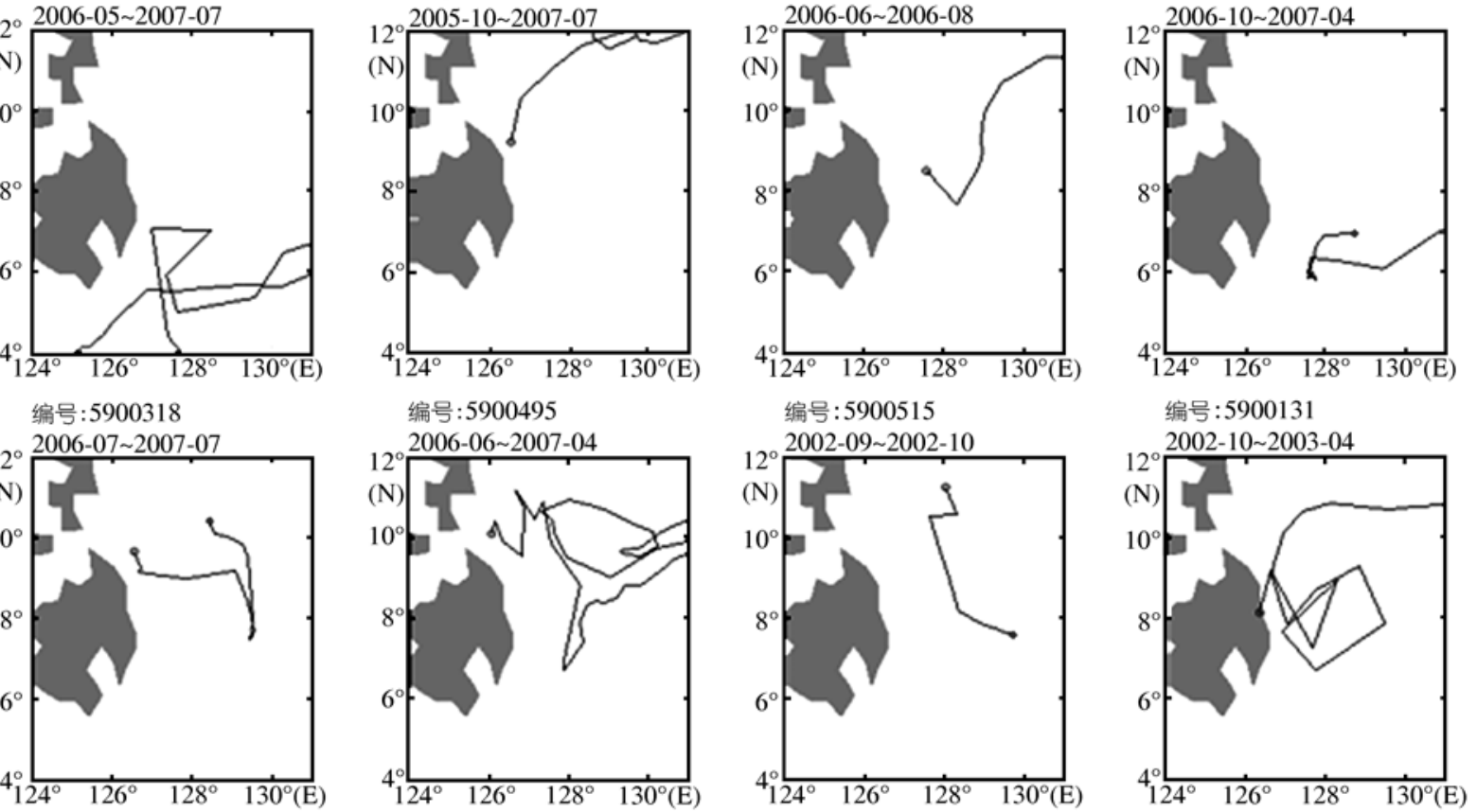

图 32003 年 1 7 月期间在棉兰老岛以东漂移的浮标轨迹

星号和圆圈分别代表每个浮标的起点和终点. 每个浮标的编号以及进入该区域的时间标注于图上方

\subsection{4 号浮标}

5900224 号浮标于 2003 年 1 月投放于棉兰老岛 以东海域, 其漂移深度为 $1500 \mathrm{~m}$ (图 4(a), 表 1)。该浮 标在入海后的 $60 \mathrm{~d}$ 内完成了两个封闭环的运动轨迹, 表明它被中层的一个浴旋所捕获(图 4(a)). 图 4 中浮 标轨迹显示在 2003 年 1 3 月(图 4(b)), 浮标在入海后 先向西南方向运动然后沿着顺时针方向做旋转运动, 完成了以 $8.8^{\circ} \mathrm{N}, 128^{\circ} \mathrm{E}$ 为中心的第一个封闭环. 在 2003 年 4 7 月, 浮标继续沿着顺时针方向运动完成 了第 2 个封闭环, 其中心位于 $9^{\circ} \mathrm{N}, 127.5^{\circ} \mathrm{E}$ (图 4(c)). 在 2003 年 8 10月期间, 浮标沿着 $127^{\circ} \mathrm{E}$ 经线向北运 动到 $10^{\circ} \mathrm{N}$ 而后又折向南运动(图 4(d)).

\subsection{1 号浮标}

5900131 号浮标于 2002 年 2 月投放于 $11.0^{\circ} \mathrm{N}$, $145.3^{\circ} \mathrm{E}$ 附近, 其漂移深度为 $1000 \mathrm{~m}$ (图 5, 表 1). 该浮 标共观测了 22 个温盐剖面, 到 2002 年 10 月 4 日, 浮 标位于 $10.9^{\circ} \mathrm{N}, 133.4^{\circ} \mathrm{E}$ 处，随后由于技术原因，浮标 在上浮过程中没有再进行温盐观测, 但是仍然自动上 浮到海面通过卫星进行定位，并下潜到漂移深度继续 漂移。该浮标在 2003 年 1 月 24 日到 4 月 16 日期间，也 就是与 5900224 号浮标观测的同一时间, 其运动轨迹 也呈现出与 5900224 号浮标运动轨迹相似的封闭环的 形态(图 5), 从而进一步表明在浮标观测期间棉兰老 岛以东确实存在着一个中层 $(1000$ 2000 m)反气旋浴. 

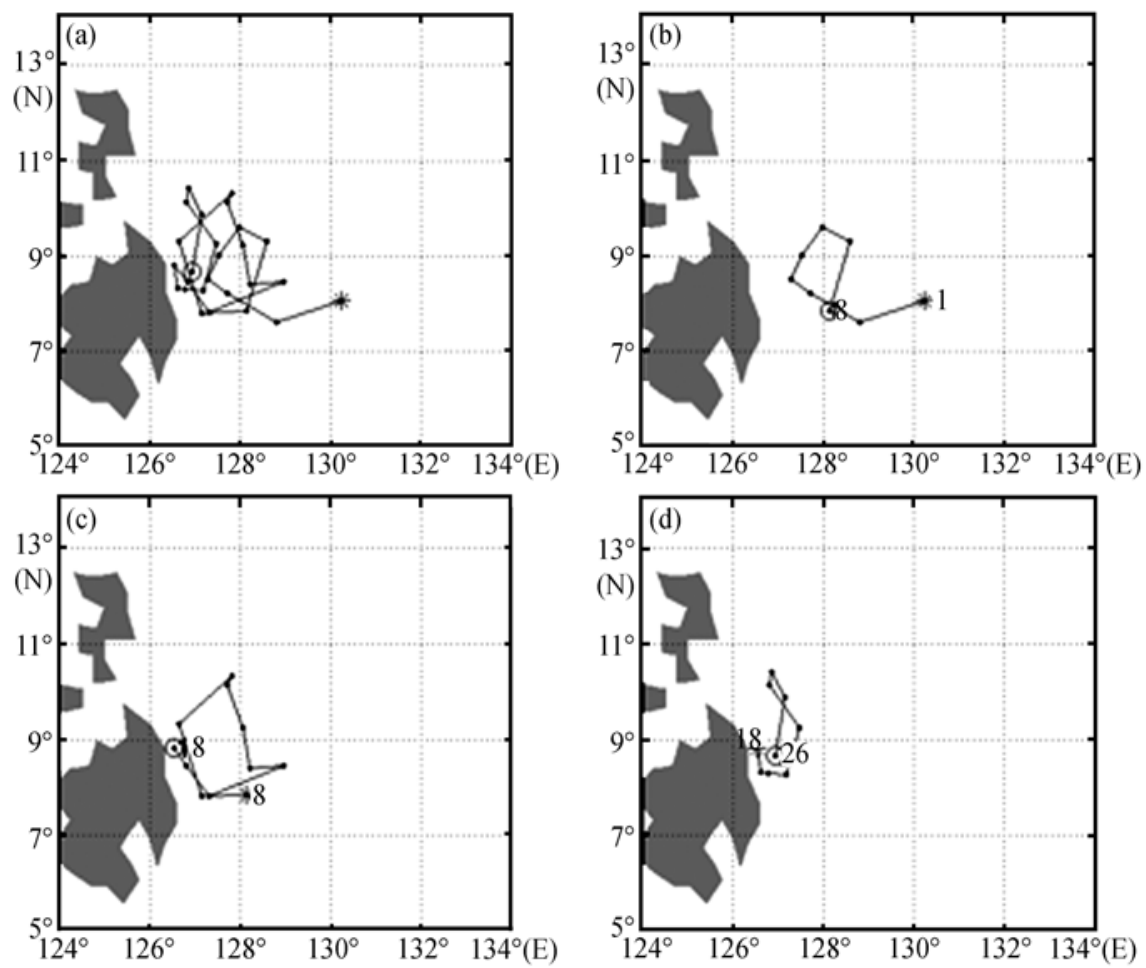

图 45900224 号浮标漂移轨迹

(a) 浮标自 1 26 号剖面轨迹(2003 年 1 10 月); (b) 浮标 1 8 号剖面轨迹(2003 年 1 3 月); (c) 浮标 8 18 号剖面轨迹(2003 年 4 7 月); (d) 浮标 18 26 号剖面轨迹(2003 年 8 10 月). 图中数字代表剖面序号, 点代表每个剖面的位置. 星号和圆圈分别代表起点和终点

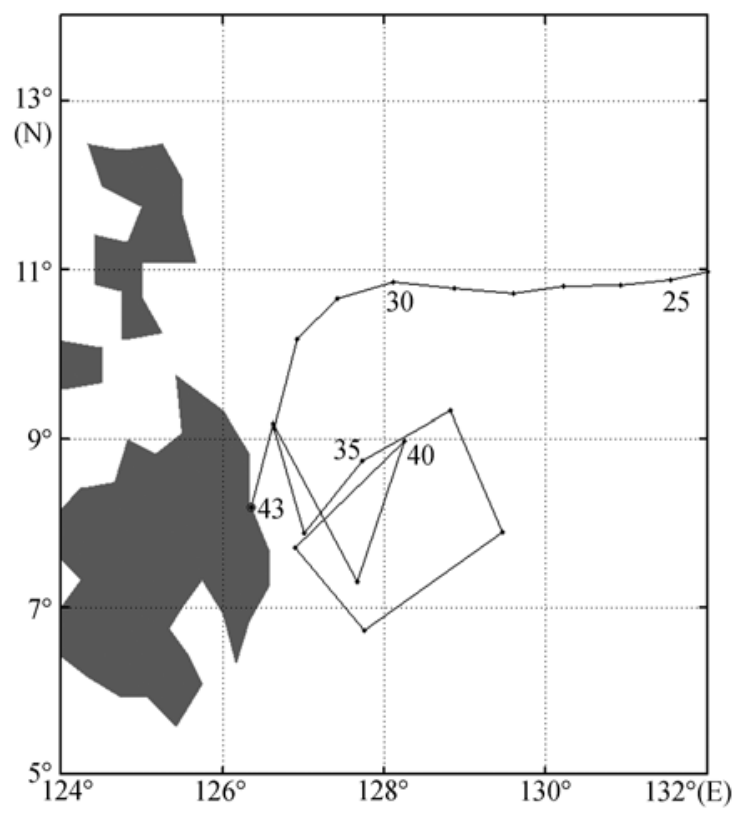

图 55900131 号浮标轨迹

数字代表浮标序号, 点代表每个剖面的位置

\subsection{6 号浮标}

2900516 号浮标的轨迹显示出比较复杂的环流 形态. 该浮标于 2005 年 10 月投放在 $13^{\circ} \mathrm{N}, 133^{\circ} \mathrm{E}$ 附 近(图 6(a), 表 1), 之后浮标慢慢向西漂移. 在 2006 年 7 8 月期间, 浮标在吕宋岛以东约 $12^{\circ} \mathrm{N}, 127^{\circ} \mathrm{E}$ 附 近完成了一个反气旋式的旋转运动(图 6(a)). 随后, 浮标向南运动, 2006 年 11 月 6 日至 12 月 26 日在 $9^{\circ} \mathrm{N}$ 附近做气旋式旋转运动, 之后浮标向南漂移, 在 2007 年 1 月, 浮标在棉兰老岛最南端附近做反气旋 式旋转运动, 2007 年 2 6 月, 浮标主要向北漂移(图 6(b)). 2007 年 5 月浮标于 $10^{\circ} \mathrm{N}, 128^{\circ} \mathrm{E}$ 附近完成了一 个尺度较小的反气旋式运动. 自 2007 年 6 月 14 日至 7 月 4 号, 浮标又向南运动(图 5(d)), 截止到本文撰稿 时浮标仍然在该海域附近运动.

\section{3 海流的拉格朗日观测}

根据浮标在海面的卫星定位信息可以推算出表 

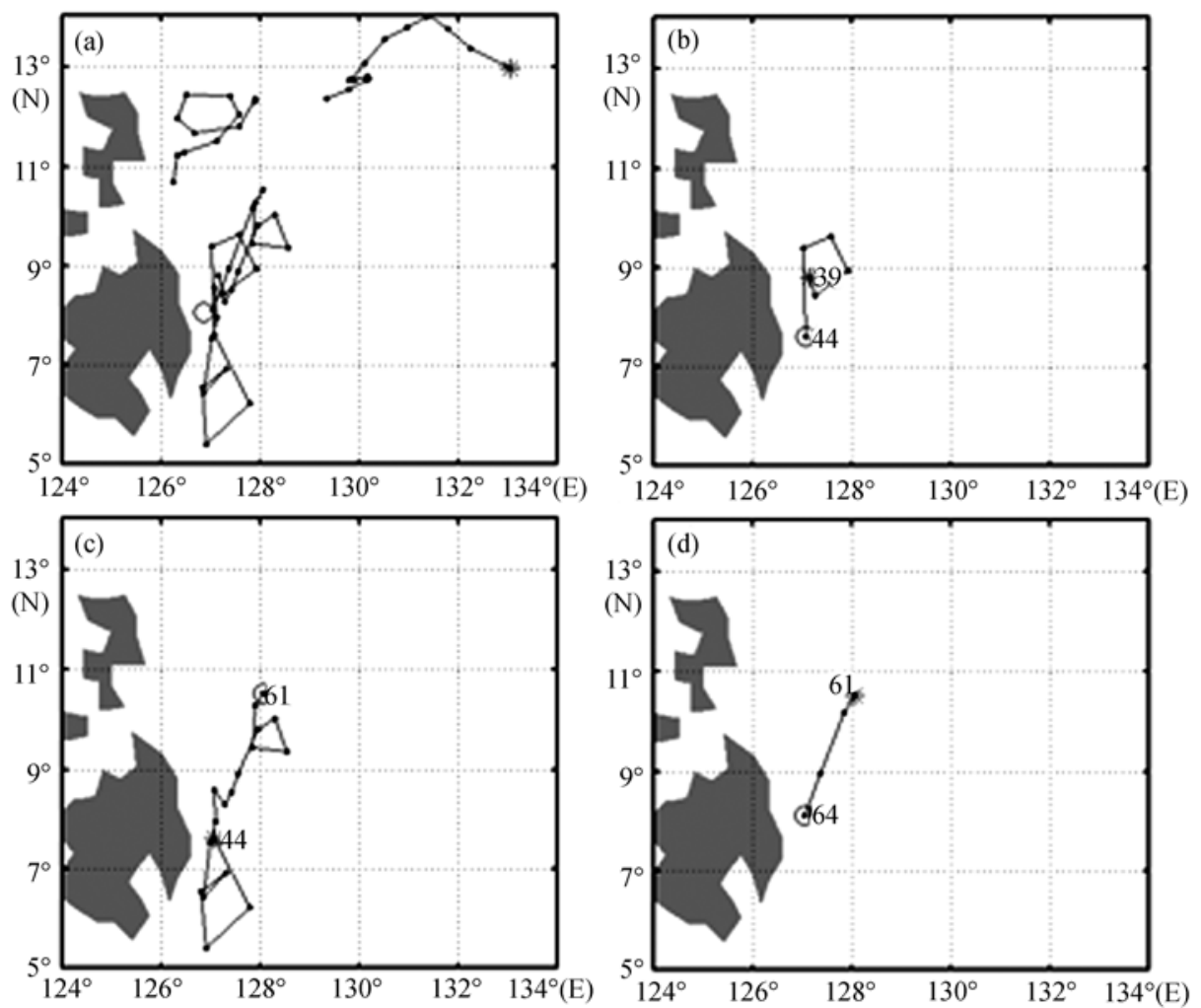

图 62900516 号浮标漂移轨迹

(a) 浮标自 1 64 号剖面轨迹(2005 年 10 月至 2007 年 7 月); (b) 浮标 39 44 号剖面轨迹(2006 年 9 月 27 日至 12 月 26 日); (c) 浮标 44 61 号 剖面轨迹(2006 年 12 月 26 日至 2007 年 6 月 14 日); (d) 浮标 61 64 号剖面轨迹(2007 年 6 月 14 日至 7 月 14 日). 图中数字代表剖面序号, 点代表每个剖面的位置. 星号和圆圈分别代表起点和终点

层流和中层(1000 2000 m)海流. 图 7 为 5900224 号浮 标在表层(图 7(a), (c), (e))和漂移深度 $(1500 \mathrm{~m}$, 图 7(b), (d), (f))上的流场分布. 上、中、下三行图分别对应的 观测时间为 2003 年的 1 3, 4 7 和 8 10 月. 图 8 为 5900131 号浮标在 2003 年 1 4 月期间表层(图 8(a)) 和漂移深度 $(1000 \mathrm{~m}$, 图 8(b))上的流场分布.

由图 7(a), (c), (e) 和 8(a) 可以看出, 这两个浮标所 反演的表层流场无论是流速大小还是流向都是非常 一致的, 均反映了非常强劲的南向流——棉兰老流, 且在 5900224 号浮标观测的 10 个月期间均非常强劲. 最强流速位于棉兰老岛沿岸且流速向南递增. 从表 层流场看来, 沿着棉兰老岛沿岸 $6^{\circ} \sim 12^{\circ} \mathrm{N}$ 之间并没有 反气旋涡存在.

浮标漂移深度上的流场结构则表现出与表层流 场截然不同的形态(图 7(b), (d), (f) 和 8(b)), 反映出该 中层流场的斜压特征. 浮标在漂移深度上所反演的 流场清晰地表明了反气旋涡的存在. 5900131 号浮标
所反演的 $1000 \mathrm{~m}$ 深度上的流速明显强于 5900224 号 浮标所反演的 $1500 \mathrm{~m}$ 深度上的流速. 两个浮标轨迹 反映的不同深度上同一个封闭环中心表明他们是受 中层深度上的同一个反气旋浴的平流作用. 5900224 号浮标在第一个封闭环中的平均切向流速为 11.9 $\mathrm{cm} \cdot \mathrm{s}^{-1}$ (图 7(b)), 在第二个封闭环中的平均切向速度 为 $12.5 \mathrm{~cm} \cdot \mathrm{s}^{-1}$, 最大流速位于 $8.4^{\circ} \mathrm{N}, 126.9^{\circ} \mathrm{E}$, 达到 $23.8 \mathrm{~cm} \cdot \mathrm{s}^{-1}$ 方向为 $13.6^{\circ}$ (正北为 $0^{\circ}$ 逆时针旋转). 5900131 号浮标的平均切向流速为 $21.1 \mathrm{~cm} \cdot \mathrm{s}^{-1}$ (图 8(b)), 大于 5900224 号浮标得到的平均流速. 从两个 浮标在漂移深度反演的流速来看, 在 2003 年 1 8 月 期间，棉兰老岛以东可能不存在跨越北赤道流的北 向的次表层海流. 不过, 在 2003 年 9 月, 5900224 号 浮标的运动轨迹似乎显示出在 $8^{\circ} \sim 10^{\circ} \mathrm{N}$ 沿着 $127^{\circ} \mathrm{E}$ 存在一支中层北向流. 但是浮标的轨迹同时表明，该 海流并未与东向流或者黑潮的深层部分相连接.

在 2006 年 9 月至 2007 年 7 月期间，棉兰老岛以 

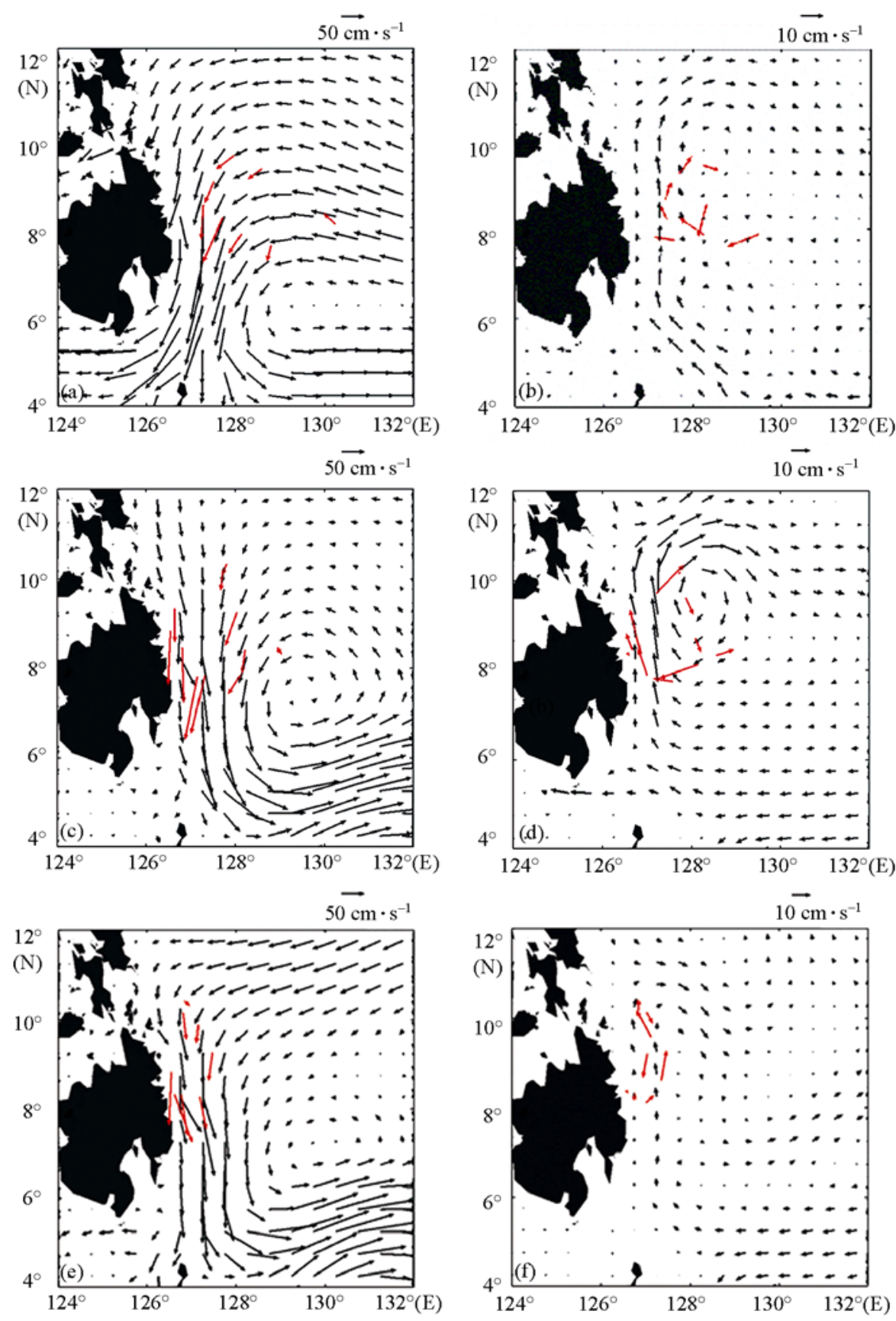

图 7 观测期间的流场分布

(a), (c), (e)为表层; (b), (d), (f)为 $1500 \mathrm{~m}$ 层. 红色粗箭头为 ARGO 浮标资料, 黑色箭头为 SODA V1.4.3 多月平均结果. (a), (b)为 2003 年 1 3 月; (c), (d) 为 2003 年 4 7 月; (e), (f) 为 2003 年 8 10 月

东的中层流先是向南流动到达 $6.5^{\circ} \mathrm{N}$ 以南, 然后又向 北流动越过 $10^{\circ} \mathrm{N}$ (图 6). 图 9 显示了 2900516 号浮标 在表层和漂移深度 $(2000 \mathrm{~m})$ 上的速度场. 图 9 的(a)和 (b), (c)和 (d), (e) 和 (f), (g) 和 (h) 对应的观测时间分别为 2006 年 7 9 月, 2006 年 10 11 月, 2007 年 1 6 月和
2007 年 6 7 月. 该浮标的表层流场再次显示了强劲 的棉兰老流(图 9(a), (c), (e), (g)). 该浮标反演的中层 流则表现为沿岸的南向流和北向流并间或几个涡旋 存在(图 9(b), (d), (f), (h)). 这些涡旋的直径通常小于 $100 \mathrm{~km}$ 且平均切向流速小于 $13 \mathrm{~cm} \cdot \mathrm{s}^{-1}$. 尽管该浮标 

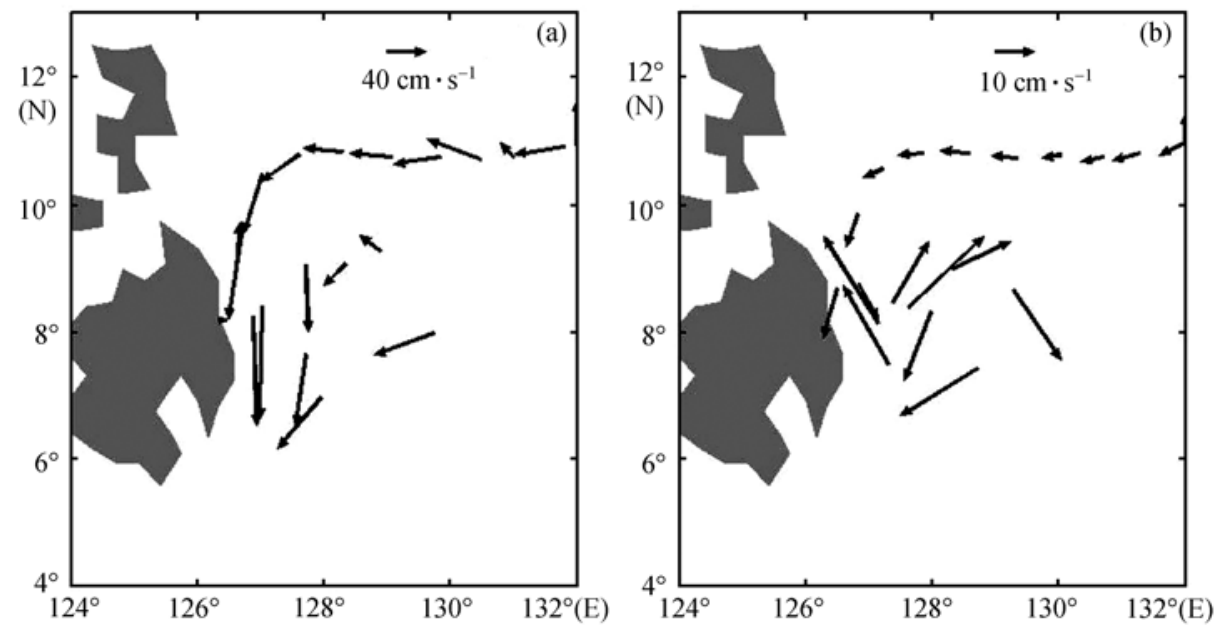

图 8 由 5900131 号浮标反演的表层(a)和漂移深度上 $(1000 \mathrm{dbar},(\mathrm{b}))$ 的流速分布
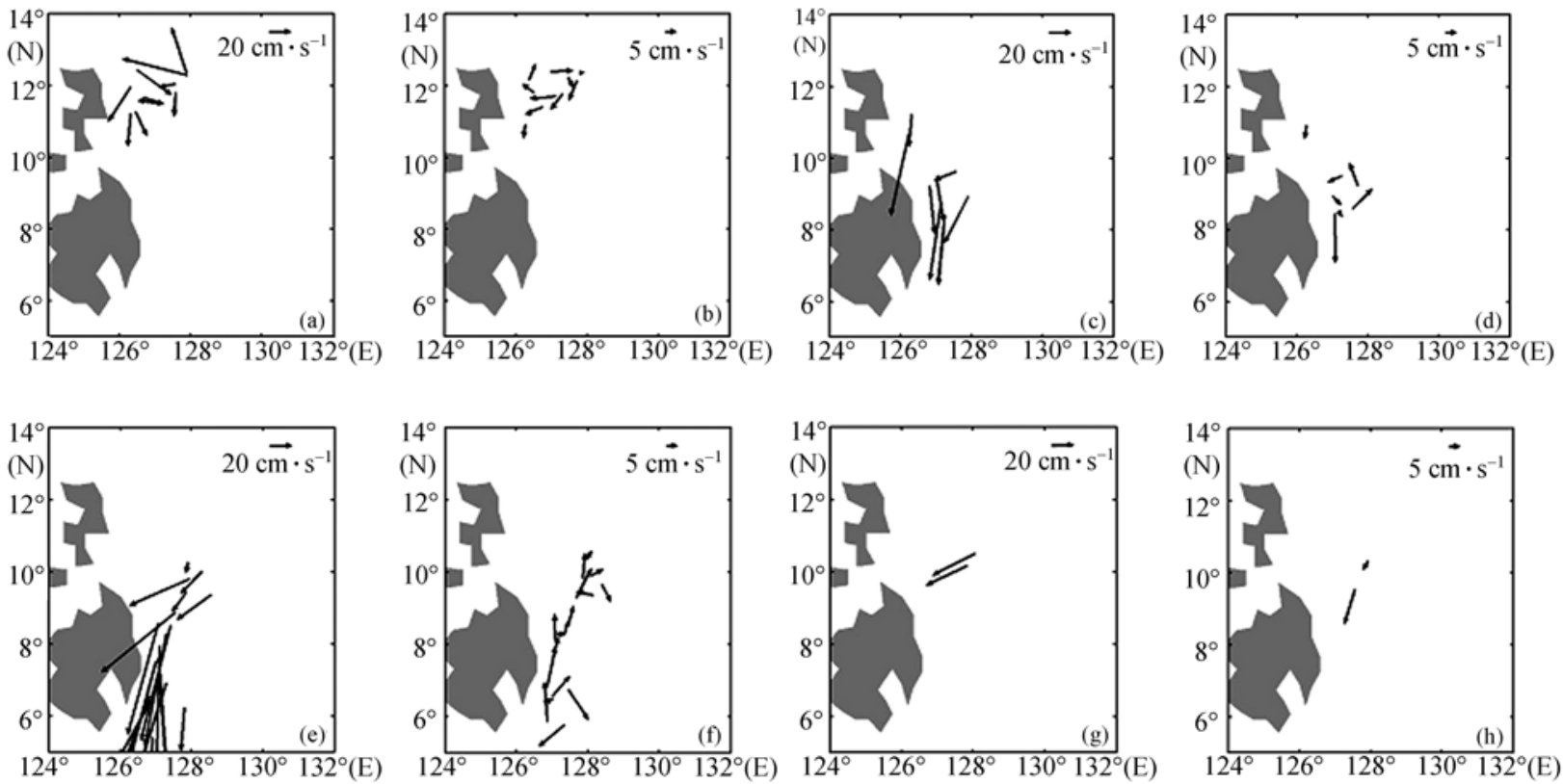

图 92900516 号浮标反演的表层流场((a), (c), (e), (g))和漂移深度流场 $(2000 \mathrm{dbar} ;(\mathrm{b}),(\mathrm{d}),(\mathrm{f}),(\mathrm{h}))$

(a), (b)为 2006 年 6 月 19 日至 9 月 27 日期间由 25 号剖面到 35 号剖面得到的流场; 图(c), (d)为 2006 年 9 月 27 日至 12 月 26 日期间由 35 号 剖面到 44 号剖面得到的流场; (e), (f)为 2006 年 12 月 26 日至 2007 年 6 月 14 日期间由 44 号剖面到 61 号剖面得到的流场; (g), (h)为 2007 年 6 月 14 日至 2007 年 7 月 14 日期间由 61 号剖面到 64 号剖面得到的流场

在 2007 年 6 月 4 14 日期间向北漂移至 $10^{\circ} \mathrm{N}$ 以北，但 是并未继续向北运动进入黑潮下层的中层流或者向 东运动进入北赤道流下面的中层流.

由于存在着西边界流，该区域垂向地转剪切是 比较强的. 因此, 必然存在这样一个疑问, 即本文中 由浮标轨迹数据估算的中层海流是否包含强劲的表 层流信息？对于 5900224 号浮标，最大上升周期为
$6.5 \mathrm{~h}$ 最大深度为 $2000 \mathrm{~m}$. 假定棉兰老流上层 $300 \mathrm{~m}$ 的平均速度为 $1 \mathrm{~m} \cdot \mathrm{s}^{-1}$, 由棉兰老流引起的最大漂移 距离为 $L_{\mathrm{dis}}$ :

$$
\begin{gathered}
L_{\mathrm{dis}}=1 \mathrm{~m} \cdot \mathrm{s}^{-1} \times 300 \mathrm{~m} / 2000 \mathrm{~m} \times 6.5 \mathrm{~h} \times 60 \mathrm{~min} \cdot \mathrm{h}^{-1} \times \\
60 \mathrm{~s} \cdot \mathrm{min}^{-1}=3.5 \mathrm{~km} .
\end{gathered}
$$

由于重力作用，浮标的下降周期通常短于上升 周期. 因此, 在浮标上升和下降过程中由于速度剪切 
所引起的最大漂移距离小于 $7 \mathrm{~km}$, 远小于浮标在漂 移深度的移动距离 $100 \mathrm{~km}$. 因此, 中层流的计算中 由表层流漂移引起的误差是可以忽略的. 然而目前 漂移到棉兰老岛以东的 Argo 浮标个数还比较少, 因 此尚不能确切的建立起棉兰老岛以东的北向中层流 与 $10^{\circ} \mathrm{N}$ 以北以及 $130^{\circ} \mathrm{E}$ 以东的其他中层流系统之间 的联系.

5900224 号浮标, 5900131 号浮标以及 2900516 号浮标轨迹以及漂移深度上的拉格朗日流表明, 棉 兰老岛以东的中层环流时空变化性很大.

Argo 浮标观测表明棉兰老岛以东中层环流 (1000 2000 m) 存在着比较活跃的中尺度涡. 根据 5900224 号浮标和 5900131 号浮标观测的反气旋浴的 平均浴度分别为 $2.4 \times 10^{-6}$ 和 $2.7 \times 10^{-6} \mathrm{~s}^{-1}$. 我们还计 算了两个浮标观测的浴旋的赤道 $\beta$ 平面 Rossby 数, 该参数的定义为 $R_{\beta}=U / \beta L^{2}, \mathrm{U}$ 和 L 分别为旋转速度和 浴旋半径 ${ }^{[17]}, R_{\beta}$ 反映了相对浴度与行星浴度的比值. $R_{\beta}<1$ 表明浴旋会因局地位浴梯度受到行星浴度梯度 $(\beta)$ 的控制而容易转变为 Rossby 波. $R_{\beta} \sim \mathrm{O}(1)$ 或者 $R_{\beta}>$ 1 表明浴旋可以长距离的进行质量和浴度的输送. 5900224 号浮标和 5900131 号浮标观测的反气旋浴的 $R_{\beta}$ 分别为 1.47 和 1.45 . 因此, 这些涡旋对于该区域的 水体交换起着一定的作用.

\section{4}

除了上述 Argo 剖面浮标的观测外, 还有几个证 据也表明在棉兰老岛以东中层深度上存在着中尺度 环流. 热带太平洋试验(Tropical Pacific Expedition, 简写为 TROPAC)期间, 在 1996 年 11 月 5 日布放的 一个沿着准等压面运动的 RAFOS 浮标(编号 175)在 棉兰老岛以东区域也被一个反气旋涡所捕获(Zenk 等 ${ }^{[18]}$ 文图 2c). 该浮标在被投放之后在 $850 \mathrm{dbar}$ 等压 面先是向西运动到达新几内亚岛附近, 随后向西北 方向运动直到被反气旋浴所捕获. 该浮标顺着反气 旋方向做旋转运动一直到 1998 年 4 月. 浮标在棉兰 老岛以东 (大约在 $7^{\circ} \mathrm{N}, 127^{\circ} \mathrm{E}$ 处)的运动轨迹清晰地显 示出与前述 Argo 剖面浮标观测相一致的中尺度浴旋 信号. 另外, Wijffels 等 ${ }^{[9]}$ 在其观测中也提到 $300 \mathrm{~m}$ 之 下存在一个暖涡信号 (见 Wijffels 等 ${ }^{[9]}$ 图 9). 以上观测 表明, 棉兰老岛以东中层深度的中尺度浴旋是经常
出现的. Firing 等 ${ }^{[10]}$ 也指出棉兰老岛以东 400 2000 m 深度上的速度场可近似为直径约 $200 \sim 300 \mathrm{~km}$, 速度 为 $0.2 \sim 0.6 \mathrm{~m} \cdot \mathrm{s}^{-1}$ 的一系列浴旋.

图 7 给出了 5900224 号浮标观测的速度场(红色 箭头)与 SODA 资料(版本为 1.4.3)速度场(黑色箭头). 从图中可以看出, 浮标观测结果和 SODA 资料(图 7(a)，(c)，(e))得到的表层流场无论是流速大小还是流 向都非常一致, 主要体现了强劲的棉兰老流. 在漂移 深度(图 7(b), (d), (f))上，两种资料所显现的流场也比 较一致, 在图 7(b)和(d)中, SODA 资料中清晰的显示 出一个反气旋浴, 其中心位于 $9.7^{\circ} \mathrm{N}, 128.2^{\circ} \mathrm{E}$, 与 Argo 浮标观测类似, 只是 SODA 资料中显示的反气 旋浴中心比 Argo 浮标观测的偏北且浴旋直径偏大. Argo 浮标观测的第二个封闭流环的直径和切向流速 均比第一个流环增大，也显示出与 SODA 资料相一 致的流态

另外, 图 7 中 SODA 资料中可以清晰地显示出棉 兰老潜流，其强度随时间变化，反气旋涡似乎是棉兰 老潜流的再循环. 但是 Argo 浮标的观测以及 RAFOS 浮标观测表明棉兰老潜流更像是反气旋浴的一个西 侧分支. 由于该区域浴旋活动非常活跃，因此浴旋的 活动可能对于该区域不同水团的混合起着重要作用. 在西边界多变的流态可能会产生南半球中层水团向 北的浴旋通量. 反气旋浴的产生机制以及他们在水 团交换中起到的作用值得进一步的研究.

\section{5}

根据 Argo 剖面浮标对海流直接的拉格朗日观测 以及对次表层海流连续的跟踪观测, 本文研究了棉 兰老岛以东中层深度上的中尺度环流. 资料分析表 明棉兰老岛以东中层环流的时空变化性极大, 并具 有显著的中尺度信号特征. 浮标轨迹显示棉兰老岛 以东中层深度 $(1000$ 2000 m) 存在着非常活跃的浴旋 运动, 这些涡旋在 $2000 \mathrm{~m}$ 深度上的平均切向速度大 约为 $10 \mathrm{~cm} \cdot \mathrm{s}^{-1}$, 在 $1000 \mathrm{~m}$ 深度上则超过 $20 \mathrm{~cm} \cdot \mathrm{s}^{-1}$. 因此, 基于参考面零流速假设的地转流计算可能会 因该区域中层深度上活跃的浴旋运动而含有较大误 差.

本文分析的 3 个 Argo 剖面浮标的观测结果表明, 2003 年棉兰老流之下存在着一个深层反气旋涡. 该 浴旋中心位于棉兰老岛以东约 $18.9^{\circ} \mathrm{N}, 127.5^{\circ} \mathrm{E}$ 处，位 
于 $1000 \mathrm{~m}$ 之下，水平尺度约 100 200 km. 在 $1000 \mathrm{~m}$ 着深度增加流速减弱, 到 $1500 \mathrm{~m}$ 深度流速减弱为 12 深度上，该浴旋的平均切向流速为 $20 \mathrm{~cm} \cdot \mathrm{s}^{-1}$, 并随 $\mathrm{cm} \cdot \mathrm{s}^{-1}$.

致谢本文所使用的 Argo 资料下载自国际 ARGO 计划(http://www.argo.ucsd.edu/, http://www.argo.org.cn/), 中国 Argo 实时资料中心在本文研究初期提供技术支持, 刘增宏以及 M. Lankhorst 提供建议及帮助, 在此一并致 谢.

\section{参考文献}

1 Toole J M, Millard R C, Wang Z, et al. Observations of the Pacific North Equatorial Current bifurcation at the Philippine Coast. J Phys Oceanogr, 1990, 20: 307-318

2 Lukas R B, Firing E, Hacker P, et al. Observations of the Mindanao Current during the Western Equatorial Pacific Ocean circulation study. J Geophys Res, 1991, 96: 7089-7104

3 Qiu B, Joyce T M. Interannual variability in the mid and low-latitude western North Pacific. J Phys Oceanogr, 1992, 22: 1062-1079

4 Fine R, Lukas R, Bingham F, et al. The western equatorial Pacific: a water mass crossroads. J Geophys Res, 1994, 99: 25063-25080

5 Wyrtki K. Physical oceanography of the southeast Asian waters. NAGA Report 2, Scripps Institute of Oceanography, University of California, San Diego, La Jolla, CA, 1961. 195

6 Hu D, Cui M, Qu T D, et al. A subsurface northward current off Mindanao identified by dynamic calculation. In: Takanao K, ed. Oceanography of Asian Marginal Seas. New York: Elsevier, 1991. 359-365

7 Wang F, Hu D X. Dynamic and Thermohaline properties of the Mindanao Undercurrent. Part II: Thermohaline structure. China J Oceanol Limnol, 1998, 16: 206-213

8 Qu T, Mitsudera H, Yamagata T. A climatology of the circulation and water mass distribution near the Philippine coast. J Phys Oceanogr, 1999, 29: 488-505

9 Wijffels S, Firing E, Toole J. The mean structure and variability of the Mindanao Current at $8^{\circ}$ N. J Geophys Res, 1995, 100: $18421-18435$

10 Firing E, Kashino Y, Hacker P. Energetic subthermocline currents observed east of Mindanao. Deep-Sea Res, Part II, 2005, 52: 605-613

11 Semtner A J, Chemin R M. Ocean general circulation from a global eddy-resolving model. J Geophys Res, 1992, 97: 5493-5550

12 Inoue M, Welsh S E. Modeling seasonal variability in the wind-driven upper-layer. J Phys Oceanogr, 1993, 23: 1411-1436

13 Xie J P, Zhu J, Xu L, et al. Evaluation of Mid-Depth currents of NCEP reanalysis data in the Tropical Pacific using Argo float position information. Adv Atmos Sci, 2005, 22: 677-681

14 Ichikawa Y, Takatsuki Y, Mizuno K, et al. Estimation of drifting velocity and error at parking depth for the Argo float. Kaiyo Kagaku Gijutsu Senta Shiken Kenkyu Hokoku, 2001. 4481-4489

15 Park J J, Kim K, King B A, et al. An advanced method to estimate deep currents from profiling floats. J Atmos Ocean Tech, 2005, 22: 1294 $-1304$

16 Machín F, Send U, Zenk W. Intercomparing drifts from RAFOS and profiling floats in the deep western boundary current along the Mid-Atlantic Ridge. Sci Mar (Barc), 2006, 70: 1-83

17 Pedlosky J. Ocean Circulation Theory. Berlin: Springer Verlag, 1996. 321-322

18 Zenk W, Siedler G, Ishida A, et al. Pathways and variability of the Antarctic Intermediate Water in the western equatorial Pacific Ocean. Prog Oceanol, 2005, 67: 245-281 